\title{
DESCRIPTION OF A SET OF MAGNETOGRAPHS DESIGNED BY W. WATSON. \\ W. Watson.
}

Some time ago I designed a set of magnetographs to be used in determining the disturbances produced by an electric railway on the three magnetic elements, the experiments being made with the view to the framing of regulations for the protection of the English magnetic observatories. Since observations had to be made at a number of stations, and accommodation for the instruments was often very rough, generally stables and sheds, it was essential that the instruments should be easily and rapidly set up and adjusted,

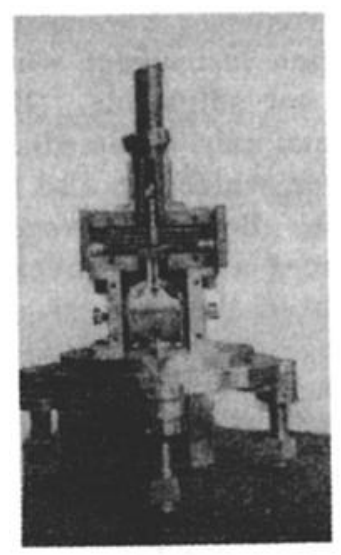

FiguRe 1.-Horizontal, INThNSity Magnetometer.

also that they should not require a darkened room. Captain Fraser, the director of the magnetic survey of India, having seen these instruments, asked me to design a set on similar lines for use at the four base stations of that survey, and although I can not claim any novelty for the arrangement as a whole, yet it has been suggested to me that a short description of the instruments might be of use to others who have to design such instruments.

No attempt is to be made in the case of the Indian magnetic survey to keep a record of the changes in the vertical component, and thus in the attached photographs, which were taken from the Indian instruments, only the declination and horizontal intensity 187 
instruments are shown. Some photographs of the vertical intensity balance used in England have, however, been added.

The declination and horizontal intensity instruments are similar, except that the suspension in the declination instrument is a fine strip of phosphor bronze, and that in the horizontal instrument is a quartz fiber. Figure I represents the horizontal intensity instrument as seen from behind, when the back part of the metal case is removed. The magnet system consists of nine small magnets cemented in an aluminium center-piece. This center-piece hooks on to the suspension and carries the movable mirror, slung on a small cross bar fixed just below the magnets. Two screws with $\mathrm{V}$-grooves in their heads rest on this cross bar, so that by screwing one in and the other out, it is possible to rotate the mirror through a small angle about a vertical axis with reference to the magnetic axis of the magnet. This adjustment is necessary in the case of the declination instrument where the azimuth of the axis of the magnet is not adjustable. The mirror can also be adjusted about a horizontal axis. Immediately below the movable mirror is the fixed mirror, which can also be adjusted about a vertical and a horizontal axis. Both mirrors are plane, and immediately in front of them is placed a plano-convex lens of $165 \mathrm{~cm}$. focal length. The total weight of the magnets and mirror, etc., is about 6.5 grams. The magnet swings inside a massive block of copper. This copper acts as a damper, and also helps to secure uniformity in the temperature of the magnet. In the case of the $H$ instrument the bulb of a thermometer projects into the cavity in which the magnet swings.

The advantage of using a number of parallel magnets in place of a single thick magnet is that, for a given magnetic moment, the weight is less, as is also the temperature coefficient.

The recording part of the instruments is shown in Figures 2 and 3 . The drums are fitted in wooden boxes, which can be easily lifted out so that the drums may be taken away to a dark room for the removal of the sensitive paper. One of the boxes containing a drum is shown at $A$, Fig. 2. The $H$ drum was removed when this photograph was taken. The clock used to drive the drums and make the time scale is a pendulum one beating seconds, and is shown at $C$. A cam attached to the hour arbor of the clock works two levers which carry two small metal flags (A, Fig. 3) that drop into the position shown in the figure for two minutes each hour and thus cut off the light which is reflected from the fixed mirror, in this way giving the time scale. 


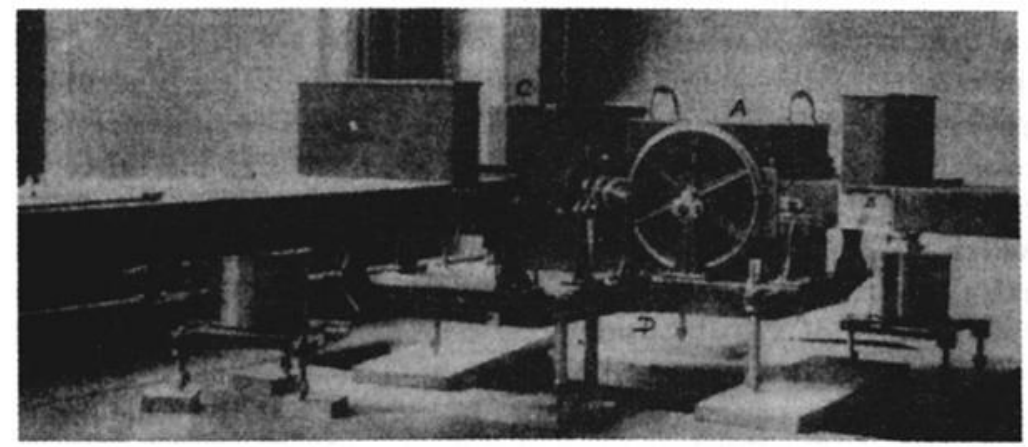

Figure 2.-General View of Magnetographs.

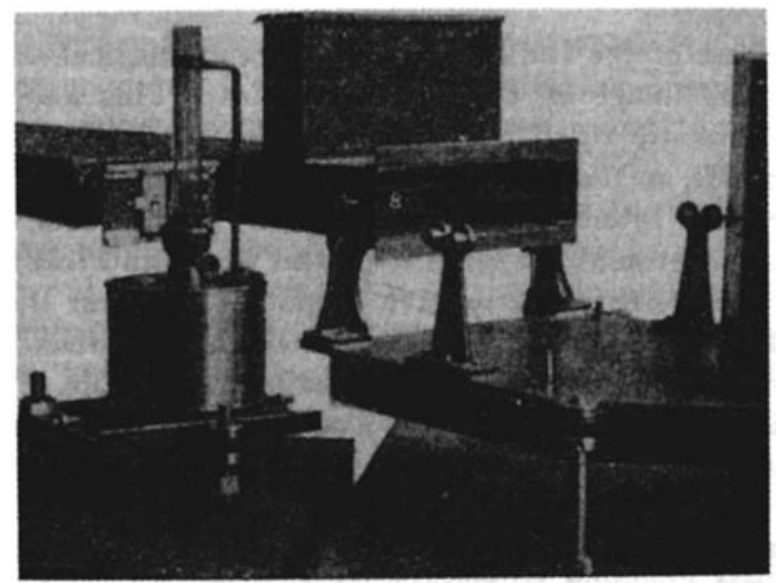

Figure 3.-EMLARGed VIFw. 
The drums are connected to the instruments by means of metal tubes, the ends of which are shown in Figures 2 and 3 . These tubes carry the slits, the cylindrical lenses $B, F i g .3$, and an arrangement for taking eye observations. In order to allow of eye observations being made, as well as to be able, without removing the drums, to see whether the spots of light are all right-i. $e$., to make certain the lamps, slits, and the mirrors of the instruments are in adjustment-a plane mirror, which stretches right across the tube in front of the cylindrical lens, is provided. This mirror can turn about a horizontal axle and is attached to the lever $B$, Fig. 2 . When this lever is turned down against a stop, the mirror is inclined at $45^{\circ}$ to the horizon, and the light reflected from the fixed and movable mirrors of the instrument, instead of striking the cylindrical lens, is reflected upwards on to a ground-glass scale fixed to the top of the upright portion of the tube ( $C$, Fig. 3 ). When the lever $B$ is turned up into the position shown the mirror closes the lower end of this upright portion of the tube and thus prevents stray light getting to the inside of the tube, and thus to the drums.

Both the slits and the lamps can be noved throtigh a distance of $3 / 4$ inch, the extent of their movement being regulated by adjustable stops. In this way two days' trace can be taken on each sheet.

In the case of the Indian instruments a cylindrical lens is nsed to reduce the image of the slits to a point. This was necessary since, for climatic reasons, it was considered unadvisable to use sensitive paper, so that without the cylindrical lenses the light was not sufficiently intense. In the English instruments, however, ordinary rapid bromide paper is used and the cylindrical lenses are dispensed with. In order to give a spot of light on the drum a horizontal slit is attached to the box which surrounds the drum and placed as near the surface of the paper as possible. The trace obtained in this way is appreciably sharper than that obtained when a cylindrical lens is used.

When a vertical intensity instrument is to be added to the others the arrangement of the clock is slightly altered. Thus the portion of the base plate, which in Fig. 2 is shown cut away at $D$, supports the clock while the vertical intensity drum occupies the place where the clock is in the figure. This drum is supported on a platform just above the axle which carries the bevel wheels that drive the drums, and is driven by a third bevel wheel attached to this axle.

The magnet of the vertical intensity balance is shown in. Fig. 4. 
It consists of eight steel rods each Io $\mathrm{cm}$. long and $0.2 \mathrm{~cm}$. in diameter. The knife edge and the movable mirror are attached to the center of an aluminium frame which carries the magnets. This frame also carries a light vaue and counterpoise which are used in adjusting the balance and sensitiveness of the magnet. Five copper plates are arranged so as to act as dampers. The magnet can be raised off the plane by means of a lever which carries a hole-slot and plane arrangement that insures the magnet always being lowered in exactly the same place.

A zinc compensating bar $A$, Fig. 4 , is used to make the readings almost, if not quite, independent of changes in temperature. The

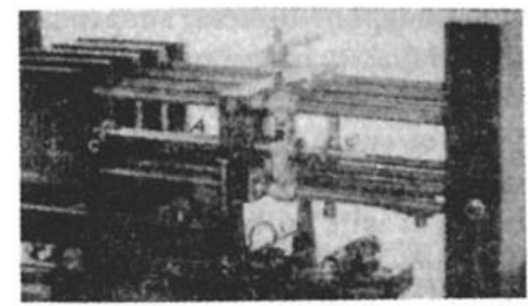

FigURE 4--Vertical INTENSITy Magnetometer.

zinc rod is screwed into an upright bar $B$ which is clamped onto two of the magnets. Two small brass weights, $C$ and $C^{\prime}$, can be screwed along the zinc rod. The zinc rod passes through a wide hole in the central aluminium frame and does not touch this frame. When adjusting the temperature compensation the two weights $C$ and $C^{\prime}$ are placed at about half a centimeter from the two ends of the zinc rod and then the rod is screwed through the upright $B$ till the compensation is nearly complete. The final adjustment is obtained by screwing the two weights $C$ and $C^{\prime}$ either both towards the upright $B$ or both away from this upright. In this way it is fairly easy to get the compensation so complete that when the temperature changes by $15^{\circ} \mathrm{C}$. the reading of the balance does not alter by $5 \gamma$.

The temperature effect in the horizontal intensity instrument is the chief objection to the use of the quartz thread unifilar magnetometer. Unfortunately, unlike most materials, fused quartz becomes stiffer as the temperature rises. As a rise of temperature is accompanied by a decrease in the magnetic moment of the magnet both effects conspire, so that the temperature coefficient of the 
instrument is the sum of the temperature coefficients of the magnet and of the rigidity of quartz. Since I have found that the temperature coefficient of the rigidity of phosphor bronze is 0.0003 , the wire getting weaker as the temperature gets greater, I was led to attempt to use a strip of this material as the suspension in a unifilar horizontal intensity magnometer. I was unable to make a satisfactory trial in the laboratory, since a neighboring electric railway caused so inuch disturbance, but owing to the kindness of the director, Dr. Glazebrook, and of Dr. Chree, I was allowed to set up two similar unifilar $H$ magnetographs, one having a quartz and the other a phosphor-bronze suspension, at the Kew Observatory. It was then found that, as expected, the temperature coefficient of the instrument with the phosphor-bronze suspension was very much smaller than that of the quartz instrument. It was, however, found that there was a creep of the spot due to the fatigue of the metal strip. This effect was of such a magnitude that whenever there is any possibility of protecting the instrument from large and rapid changes of temperature it will be better to use a quartz suspension and correct the readings for changes of temperature. This, of course, involves having a recording thermometer of some kind placed alongside the magnetometer. Circumstances may, however, arise in which a record is required for a comparatively short time and during which time rapid changes of temperature may take place, in which case a phosphor-bronze suspension may be found of use. In this connection it may be pointed out that the temperature coeffcieut of such a bundle of magnets as is employed in these instruments depends to a great extent on the number and nearness of the magnets. Hence, by varying the number of magnets we can adjust our temperature coeficient to suit that of the suspension. 